\title{
Current Opportunities and Challenges in Biopolymer Thin Film Analysis-Determination of Film Thickness
}

\section{OPEN ACCESS}

Edited by:

Florent Allais,

AgroParisTech Institut des Sciences et Industries du Vivant et de L'environnement, France

Reviewed by:

Maria Lucia Caetano Pinto Da Silva,

University of São Paulo, Brazi

Céline Moreau,

U1268 Biopolymères, Interactions, Assemblages (BIA), France

*Correspondence: Tiina Nypelö tiina.nypelo@chalmers.se

Specialty section:

This article was submitted to Chemical Reaction Engineering, a section of the journal Frontiers in Chemical Engineering

Received: 08 August 2021 Accepted: 16 September 2021 Published: 21 October 2021

Citation: Spirk S, Palasingh $C$ and Nypelö $T$ (2021) Current Opportunities and Challenges in Biopolymer Thin Film Analysis-Determination of

Film Thickness.

Front. Chem. Eng. 3:755446. doi: 10.3389/fceng.2021.755446

\author{
Stefan Spirk ${ }^{1}$, Chonnipa Palasingh ${ }^{2}$ and Tiina Nypelö ${ }^{2,3 *}$ \\ ${ }^{1}$ Institute of Bioproducts and Paper Technology, Graz University of Technology, Graz, Austria, ${ }^{2}$ Applied Chemistry, Department of \\ Chemistry and Chemical Engineering, Chalmers University of Technology, Gothenburg, Sweden, ${ }^{3}$ Wallenberg Wood Science \\ Center, Chalmers University of Technology, Gothenburg, Sweden
}

Polymer thin films with thickness below $100 \mathrm{~nm}$ are a fascinating class of 2D materials with commercial and research applications in many branches ranging from coatings to photoresists and insulating materials, to mention just a few uses. Biopolymers have extended the scope of polymer thin films with unique materials such as cellulose, cellulose nanocrystals, cellulose nanofibrils with tunable water uptake, crystallinity and optical properties. The key information needed in thin biopolymer film use and research is film thickness. It is often challenging to determine precisely and hence several techniques and their combinations are used. Additional challenges with hydrophilic biopolymers such as cellulose are the presence of humidity and the soft and often heterogenous structure of the films. This minireview summarizes currently used methods and techniques for biopolymer thin film thickness analysis and outlines challenges for accurate and reproducible characterization. Cellulose is chosen as the representative biopolymer.

Keywords: thin films, thickness, biopolymers, analytics, cellulose

\section{INTRODUCTION}

The most relevant biopolymer used in thin film preparation is probably cellulose. Cellulose can be manufactured into thin films from solutions either by direct dissolution (Fält et al., 2004; Kargl et al., 2015; Lu et al., 2020) or by the use of soluble cellulose derivatives that are converted to cellulose after processing (Schaub et al., 1993; Kontturi et al., 2003; Kontturi et al., 2007) as well as from particle suspensions (Edgar and Gray, 2003; Kontturi et al., 2007; Ahola et al., 2008; Aulin et al., 2009; Niinivaara et al., 2016). While preparative aspects are still under scientific research (Kontturi et al., 2006; Kargl et al., 2015; Weißl et al., 2018; Weißl et al., 2019a), the past years were dominated by a quest for applications of these films in optics, catalysis, photoresists and as biosensors (Kontturi and Spirk, 2019; Raghuwanshi and Garnier, 2019). The main advantage of cellulose thin films compared to $3 \mathrm{D}$ materials is that the chemistry, morphology, and optical properties can be tuned by the preparation conditions and choice of cellulose materials. As cellulose thin films are among the most used biopolymer thin films, they are employed as the demonstrator of thickness analysis in this Minireview. However, the analytical techniques and the challenges involved are applicable to many other biopolymer films such as chitin (Kittle et al., 2012), chitosan (Spirk et al., 2013), lignin (Norgren et al., 2006; Tammelin et al., 2006), and the vastly growing field of thin films targeted for creating model biointerfaces (Raghuwanshi and Garnier, 2019) for protein cell interaction studies (Manini et al., 2020) and properties of DNA (Sun et al., 2014). 
The film thickness of thin films is often decisive for their properties. It is also required for deriving many of the essential other properties of thin films such as quantitative comparative analysis of adsorption on and swelling of the films. An inaccurate thickness value leads to discrepancy in the further calculated parameters and hence, film thickness requires accurate determination. This minireview presents the currently employed methods for polymer thin film thickness determination with focus on biopolymer thin films with thickness less than $100 \mathrm{~nm}$.

\section{BIOPOLYMER THIN FILM THICKNESS DETERMINATION}

What should be kept in mind with control and determination of thickness is that almost always it is accompanied by visualization of the film structure to judge film evenness. In addition to establishing how the appearance of the film is, indeed, knowledge of either intentional or unintentional contours and roughness on film surface is essential to accompany film thickness determination since conformation in larger length scale than the film thickness reflects on the thickness values retrieved.

\section{Visualization and Tracking of Topography on a Substrate}

Oftentimes, a high resolution electron microscope and imaging of a film cross-section can be adequate to retrieve a thickness value as demonstrated for $150 \mathrm{~nm}$ thick cellulose films (Puspasari et al., 2015). However, only a few examples are available for cross-sectional analysis of thinner biopolymer films for mere film thickness determination due to laborious sample preparation (Weißl et al., 2019b), low contrast of most of organic matter in electron microscopy, and its tendency to decompose in an electron beam. Indeed, the most used tool for a thin film thickness measurement is a stylus profilometer that tracks the surface conformation by mechanically moving a stylus across the film substrate and monitoring the force applied (Niegelhell et al., 2016b; Niegelhell et al., 2017; Weißl et al., 2018). For recording film thickness, scratching of the film on the substrate allows creation of a contour of the substrate, and the film and the height difference between the substrate and the film surface is monitored (Siderov et al., 2013). The force and material applied to scratch the film is important, as it is crucial not to indent the substrate. Another known challenge is that when the contour is analyzed by recording the morphology, contact is required with the surface in the measurement and it can destruct a soft film and alter the recorded value of thickness.

Similar to the profilometry, atomic force microscopy (AFM) is used for thickness determination by analysis of height profiles as shown for dry and for wet cellulose films (Fält et al., 2004; Ganner et al., 2015; Reid et al., 2016). Disadvantage of the scratch method here as well is the potential damaging of the substrate and hence affecting the thickness value. Indeed, when using razor blade for scratching, a 1-2 nm penetration also to the substrate when determining thickness of cellulose films, has been found (Fält et al., 2004). To circumvent this, polymer film thickness can be determined by controlling the loading force of the AFM tip on film surfaces, scratching the film with the tip and recording the height profile (Ton-That et al., 2000). Prior to extraction of the height profile from AFM analysis, it is vital to apply corrections to the image to account for the plane tilt and scanner movements (Canale et al., 2011).

\section{Sarfus Technique}

Sarfus is a setup where an optical microscope in crosspolarization mode is used in conjunction with specific substrates, so called surfs. The surfs consist of a silicon substrate with a top coating that does not polarize light on reflection and therefore leads to a signal enhancement of the film attached to that layer (Chiang and Yang, 2011). In combination with the use of a calibration standard, layer thicknesses down to $0.3 \mathrm{~nm}$ can be determined. The method can also be used in combination with scratching the surface, to further reduce potential experimental errors. An example where the method has been used to visualize biopolymer samples are chitosan-silane hybrid thin films (Figure 1) (Spirk et al., 2013). Layer thicknesses in the range between 8 and $100 \mathrm{~nm}$ were analyzed with this fast technique with high throughput.

\section{Quartz Crystal Microbalance with Dissipation Monitoring}

QCM-D is a gravimetric method to determine deposited mass on a substrate. The detection is based on a change in the resonance frequency of the sensor when its mass changes by deposition of a thin film. Furthermore, the adsorption and desorption of molecules on such films can be studied (Reviakine et al., 2011). The Sauerbrey equation (1959) (Equation 1) is used for mass $(\Delta m)$ determination for rigid films and requires input of change in frequency $\Delta f$, overtone number $n$, crystal sensor

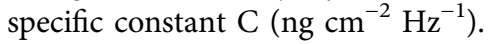

$$
\Delta m=-C \frac{\Delta f}{n}
$$

QCM-D has been utilized to determine thickness of cellulose thin films by calculating the mass of the film via Eq. 1 using the changes in frequency response before and after deposition of a cellulose layer on the QCM-D sensor (Peresin et al., 2012; Tammelin et al., 2015; Palasingh et al., 2021). This mass together with an input value of (assumed) density, $\rho$, is then used to extract film thickness (d) according to Eq. 2:

$$
d=\frac{\Delta m}{\rho}
$$

Mohan et al. (2012) used the QCM-D thickness determination method for trimethylsilyl cellulose and cellulose films accompanied by density values that were determined by X-ray reflectivity (XRR) to 1.0 and $1.5 \mathrm{~g} \mathrm{~cm}^{-3}$, respectively.

The challenge of the thickness determination using the resonance frequency before and after the film deposition is that it is sensitive to contamination that may take place when the sensor is removed, coated, and inserted again into the surface sensitive device. Another advantage of the QCM-D method is 

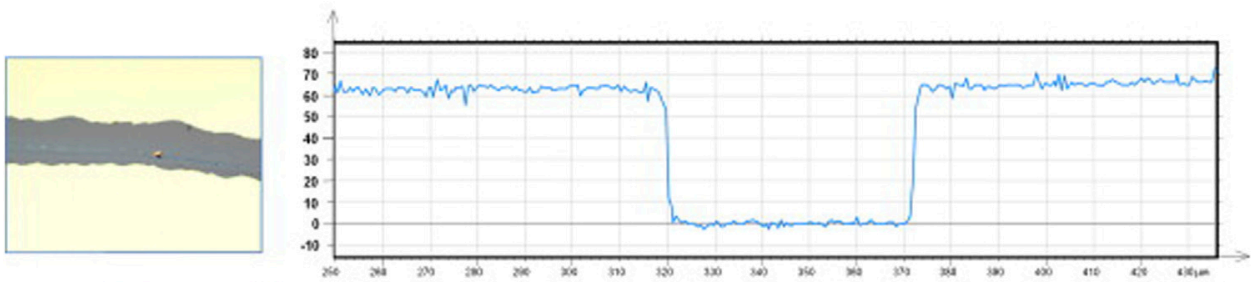

\section{Stock solution}
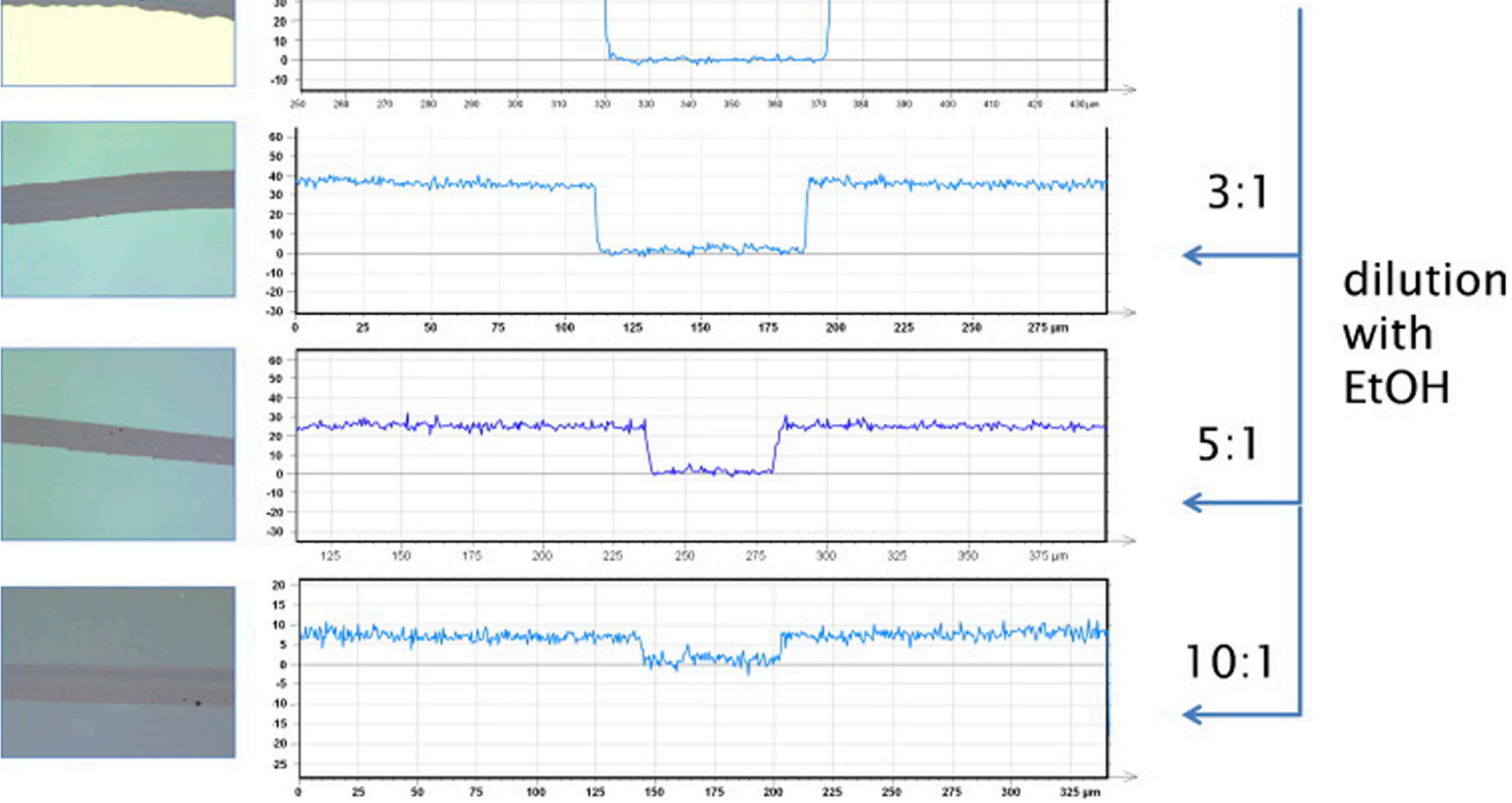

10:1

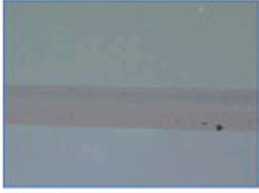

FIGURE 1 | Left: microscope images (20x magnification, $200 \times 200 \mu \mathrm{m}$ ) of chitosan-silane hybrids prepared by spin-coating. The scratch in the middle of the images has been made using a cannula. Right: height profiles (nm) of these layers determined by Sarfus. Reprinted from Spirk et al. (2013), with permission from Elsevier.

that also changes of layer thickness by swelling either via gas phase (humidity) or liquid water can be tracked (Niinivaara et al., 2016).

However, soft systems with dissipation $>0$ are sensitive to viscoelastic properties and the Sauerbrey equation is inadequate to represent the film mass. The Voigt model is a viscoelastic model applied for analysis of viscoelastic systems in QCM-D (Reviakine et al., 2011). The model accounts for viscosity and elasticity of the film through complex shear modulus (Voinova et al., 1999), which has been used in analysis of biopolymer films, for instance, xyloglucan and gums (Eronen et al., 2011), carboxymethyl cellulose (Eronen et al., 2011; Orelma et al., 2011) and chitosan (Orelma et al., 2011) on cellulose films. Further models and guideline for choosing and interpreting the data can be found in Reviakine et al. (2011) However, analysis of soft and hydrated biopolymer films using Voigt model has been shown to deviate from values from surface plasmon resonance (SPR) (Mohan et al., 2014) that is considered to be a direct analysis to gain value for film mass. Hence, the latter is often the method of choice over QCM-D when it comes to determination of thickness.

\section{Surface Plasmon Resonance Spectroscopy}

SPR is an optical technique that relies on the interaction of light with surface plasmons that are present on metal surfaces. In a typical setup, the incident light beam's angle is varied, and the intensity of the reflected light is recorded. At a specific angle, the intensity of the reflected light has a minimum as the plasmons resonate. Under resonance conditions, the surface plasmons react sensitive towards changes of their environment, i.e., permittivity of the surrounding media (e.g., solvent, film). This also applies when additional layers or molecules are added via adsorption measurements in situ as they trigger changes in the permittivity of the entire thin film assembly (Jung et al., 1998). However, as it is a surface sensitive phenomenon, typically films with layer thicknesses larger than one micron cannot be analyzed. The phenomenon can be explained by classic physics as shown in Eq. 3 where $w$ is the angular frequency, $c$ is the speed of light in vacuum, $\varepsilon_{\mathrm{o}}$ is the permittivity of refractive element (prism), $\theta_{\mathrm{c}}$ is the incident angle and $\varepsilon_{1}$ and $\varepsilon_{2}$ are the permittivity of the ambient medium and the metal surface.

$$
\frac{\omega}{c} \sqrt{\varepsilon_{0}} \sin \left(\theta_{c}\right)=\frac{\omega}{c} \sqrt{\frac{\varepsilon_{1} \varepsilon_{2}}{\varepsilon_{1}+\varepsilon_{2}}}
$$

The main information that can be extracted from such SPR curves is the refractive index and the layer thickness, with resolution limits in the submonolayer regime (Niegelhell et al., 2016a) by employing multi-layer fitting on the basis of the Fresnel equations. These fitting procedures yield a set of continuous layer thickness-refractive index pairs, whose unique solution requires either the use of a multi wavelength setup (multi-color method) 
or to investigate the film in different media [two media approach (Peterlinz and Georgiadis, 1996)]. In adsorption conditions, also the kinetics of layer growth/molecules adsorption can be observed in situ using SPR, with the advantage that the layer thickness determination is not influenced by the softness of the films. If the refractive index increment $\mathrm{dn} / \mathrm{dc}$ is known, the de Fejter equation Eq. 4 can be directly used to determine the amount of adsorbed materials $(\Gamma)$ from the shift in the surface plasmon resonance angle $(\Delta \theta)$ without applying multi-layer fitting procedure ( $\mathrm{k}$ and $d_{p}$ are instrument constants) (De Feijter et al., 1978).

$$
\Gamma=\frac{\Delta \Theta \times k \times d_{p}}{d n / d c}
$$

Via the density of the adsorbed compounds, the layer thickness can then be easily evaluated. In principle also the density can be determined at the interfaces. Sampl et al. (2019) showed that the outermost layer of cellulose thin films $(3 \mathrm{~nm})$ has a different refractive index and density than the bulk film. Combining SPR with QCM-D, swelling studies can be performed that allow the monitoring of water uptake inside different biopolymer thin films (Kontturi et al., 2013).

\section{Ellipsometry}

Ellipsometry is used to determine optical constants and thickness of a material (Azzam et al., 1978). It measures change in polarization of reflected or transmitted light. Upon reflection at a plane surface, linearly polarized radiation generally becomes elliptically polarized. Its polarization state can be described by two ellipsometry parameters: amplitude ratio $(\Psi)$ and phase shift difference $(\Delta)$ of two mutually orthogonal polarized components of the reflected waves. The change in polarization is the ellipsometry measurement, commonly written as:

$$
\rho=\tan (\psi) \mathrm{e}^{\mathrm{i} \Delta}
$$

The measured $\Psi$ and $\Delta$ cannot be converted directly into the optical constants of the material but requires modeling. Description of some models for thin biological films can be found in Arwin (2000). When a suitable model is chosen, the ellipsometry data are fitted to the model. The optical models give then the complex refractive indices $\tilde{n}$ (Eq. 5) that is expressed as the sum of $n$ and extinction coefficient $(k)$, and finally film thickness.

$$
\widetilde{n}=n+\mathrm{i} k
$$

Typically, for enabling the measurement the surfaces must be light reflecting, and the film has no (or low) light absorption or the complex refractive index of the film is known (Tengvall et al., 1998). When the refractive index of the material is known, the determination of thickness is simple (Tengvall et al., 1998; Höök et al., 2002). In some cases, when refractive index is unknown, measuring at multiple wavelengths (spectroscopic ellipsometry) can provide both refractive index and thickness. The thickness determination of thin films requires building a multilayer model that includes the substrate, e.g., silicon, silicon dioxide, biopolymer layer and air and is often performed using commercial software with embedded models and for example, using refractive indices of the known layer materials and iterative fitting [e.g., (Eriksson et al., 2007)].

\section{Reflectometry}

Reflectance using X-rays (XXR) (Foster et al., 1990; Kontturi and Lankinen, 2010), neutrons (Thomas and Penfold, 1996) and light (Buron et al., 2006; Cranston and Gray, 2008) have been used for thin film characterization, among them thickness or change in thickness. The analysis of thin films is based on an incident beam that penetrates to the sample reaching to regions that have different refractive indices, e.g., at a film-substrate interface. The beam is reflected from the interfaces and interference produces a Fresnel reflectance pattern. The ratios of heights of the maxima in the pattern relate to film thickness and interface width and hence lead to possibility to resolve film thicknesses. (Foster et al., 1990). The periodicity of the oscillation is inversely proportional to the film thickness. Lu et al. (2020) have analyzed cellulose thin films $(8-88 \mathrm{~nm})$ using XRR. X-ray reflectivity can be also assessed to observe a response of cellulose thin films upon humidity variations. In such experiments, the films are placed in humidity chambers that have windows for the X-ray beams. Figure 2 shows such results of cellulose thin films whose thickness increased from 45 to $52 \mathrm{~nm}$ when humidity increased from 0 to $70 \%$ (Figure 2A). Figure 2B shows that XRR yields also different densities for the bulk layer and the layer that is exposed to the air interface.

\section{DISCUSSION}

\section{Film Thickness Value is Essential for Determining Other Properties}

An estimation of film thickness is valuable for many purposes but an utmost necessity for some analyses. Hydration and swelling of hydrophilic biopolymers direct many of their properties and already in ambient conditions they interact with humidity. Craig and Plunkett (2003) pioneered a solvent exchange method to determine water in polyelectrolyte thin films. Kittle et al. (2011) applied the method to determine amount of water $\left(\Gamma_{\text {water }}\right)$ in cellulose thin films (Eq. 7) by exchange of water to heavy water $\left(\mathrm{D}_{2} \mathrm{O}\right)$ and utilizing the density difference for determining the amount of water in a layer, according to Eq. 8.

$$
\begin{aligned}
\Gamma_{\text {water }} & =-C\left(\frac{\Delta f}{n}\right)_{\text {water }} \\
\left(\frac{\Delta f}{n}\right)_{\text {water }} & =\frac{\left(\frac{\Delta f}{n}\right)_{f i l m}-\left(\frac{\Delta f}{n}\right)_{\text {bare }}}{\left(\frac{\rho_{\mathrm{D}_{2} \mathrm{O}}}{\rho_{\mathrm{H}_{2} \mathrm{O}} \mathrm{O}}\right)-1}
\end{aligned}
$$

Where $\rho_{D_{2} \mathrm{O}}$ and $\rho_{\mathrm{H}_{2} \mathrm{O}}$ are the densities of $\mathrm{D}_{2} \mathrm{O}$ and $\mathrm{H}_{2} \mathrm{O}$, respectively. The $(\Delta \mathrm{f} / \mathrm{n})$ water is then be used to determine the water concentration in the film. The method has recently been used for determination of water content in polyelectrolyte multilayers (Kittle et al., 2021). The film thickness affects the amount of bound water and needs to be known to enable direct quantitative comparison. Furthermore, the film 

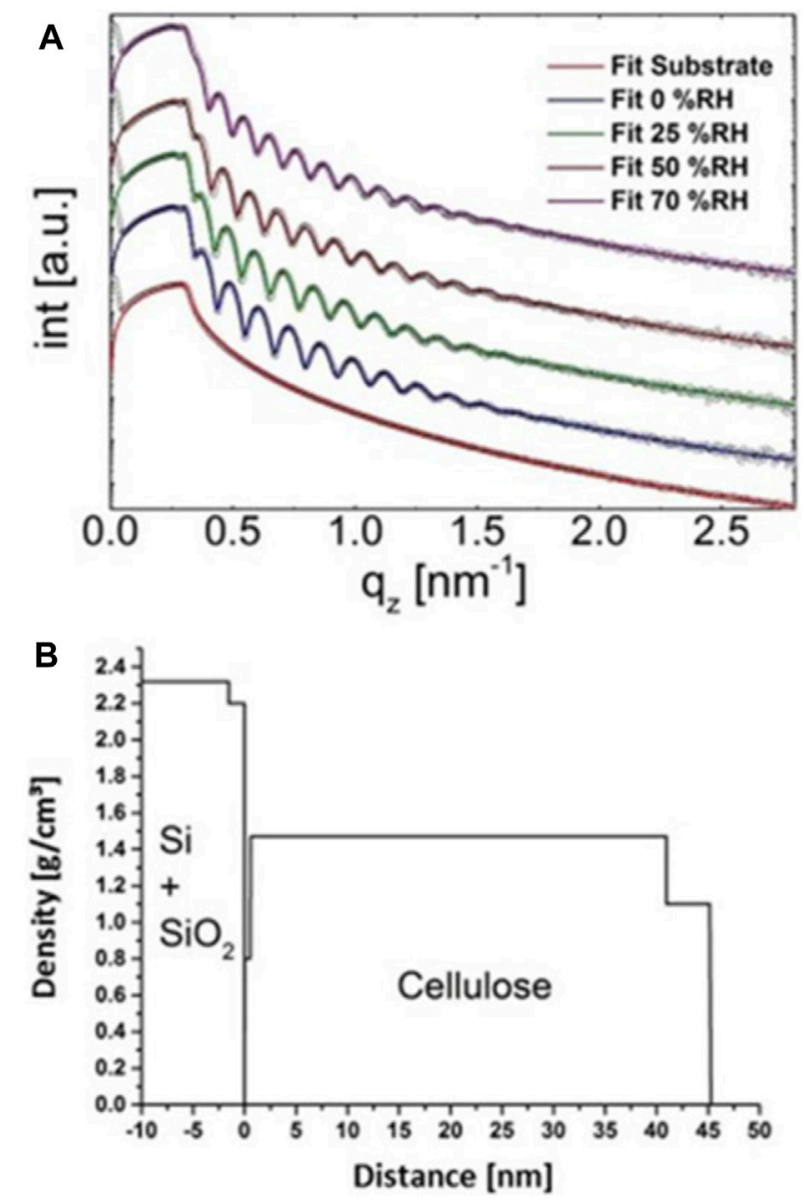

FIGURE 2 | (A) XRR curves of a cellulose thin film on a silicon water at different humidities and $(\mathbf{B})$ the resulting density profiles at $0 \%$ relative humidity. Reproduced with permission (CC BY) from Sampl et al. (2019).

thickness has been shown to affect, e.g., adsorption of xyloglucan on cellulose films (Kittle et al., 2018). Also SPR has been used for swelling determination by utilizing the inverse proportionality of volume and optical intensity (Erdoğan et al., 2008). However, also in this case, the film thickness was determined independently using ellipsometry. The power of combined methods.

The mostly used methods for determining thin film thickness are tracking conformation (profilometry, AFM), XRR, SPR, QCM-D and ellipsometry (Figure 3). Each of the method have their benefit but also a challenge. AFM and profilometry thickness determination requires scratching of the film that may lead to an artifact of also changing the substrate. The measurement itself may also be affected if the film is deformed by the stylus or AFM tip. Thickness analysis of films with QCM-D is non-destructive, however a challenge is that often the film is formed in a spin coating or other device that requires measurement of the sensor before deposition and after and is a source for an artifact for this gravimetric method. Another challenge arises from that a density value is needed for calculating thickness and in the case only an estimate is available, decreases accuracy. SPR, ellipsometry and XRR are non-destructive methods which allow for extremely precise film thickness determination $( \pm 0.1 \mathrm{~nm})$. They also provide additional information on film structure (XRR: roughness, density) and optical constants (SPR, ellipsometry: refractive indices). Their drawback is that the required modelling procedures are more laborious than, e.g., measuring a step height of a scratched surface using AFM.

The coupling of methods may give rise to additional information, particularly if, e.g., QCM-D and SPR are coupled, when exploring swollen films. This allows to determine the water content of the films. Another example was described by Gesang et al. (1995) who compared AFM and ellipsometry analysis of synthetic organic films. They pointed out that the areas investigated by ellipsometry and AFM are very different, the ellipsometry reaching about $2-3 \mathrm{~mm}^{2}$ (the exact value depending on the angle and focus of incident light), and the AFM thickness determination being local with the step height assigned to smaller areas, such as in hundreds of nanometers up to microns in frame dimensions. Discrepancy was detected above film thicknesses of $10 \mathrm{~nm}$ between ellipsometry and AFM analysis (smaller values with AFM), likely to be due to the restriction of AFM to measure large steps in height. The study used a scratched square to reveal the substrate and identified two sources of potential error: the square created by AFM tip can be that the tip cannot penetrate the film fully during scanning square formation. Other artifact can be that the tip cannot reach the bottom of the scanning square during imaging (Gesang et al., 1995). However, the AFM was rewarded to be potentially very accurate technique that does not rely on models and hence valuable for further development of models for ellipsometry. Also Mykhaylyk et al. (2007) suggest AFM techniques valuable for thickness determination, for crosschecking an ellipsometric thickness obtained from an assumed value of the thin-film refractive index, or for determination of unknown thin-film optical parameters when combined with ellipsometry measurements. They investigated polystyrene films on silicon substrates and found good agreement between AFM and ellipsometry in the $80-130 \mathrm{~nm}$ thickness range. The complementarity of ellipsometry and AFM has also been pointed out in that AFM provides the topographical details that are challenging to access with ellipsometry (Siqueira et al., 1995; Kosaka et al., 2005). Cranston and Gray (2008) compared cellulose nanocrystal film thicknesses using scratch-height analysis with AFM, ellipsometry, wavelength-dependent optical reflectometry and angle-dependent optical reflectometry and recorded $191 \pm 4,184 \pm 2,195$, and $174 \pm 11 \mathrm{~nm}$, respectively for 10 bilayers. The wavelength-dependent optical reflectometry values for film thickness were consistently larger than the other methods and was denoted to be due to the homogeneous film model that may not represent the real film configuration.

\section{The Soft and Heterogenous Biopolymer Films-An Added Challenge}

It seems that the often present structure, configuration, and roughness that lead to heterogeneous films are generally sources of uncertainty in the film thickness analysis. Some techniques are facile to identify surface roughness and thickness, e.g., AFM. While in other techniques, the film is 


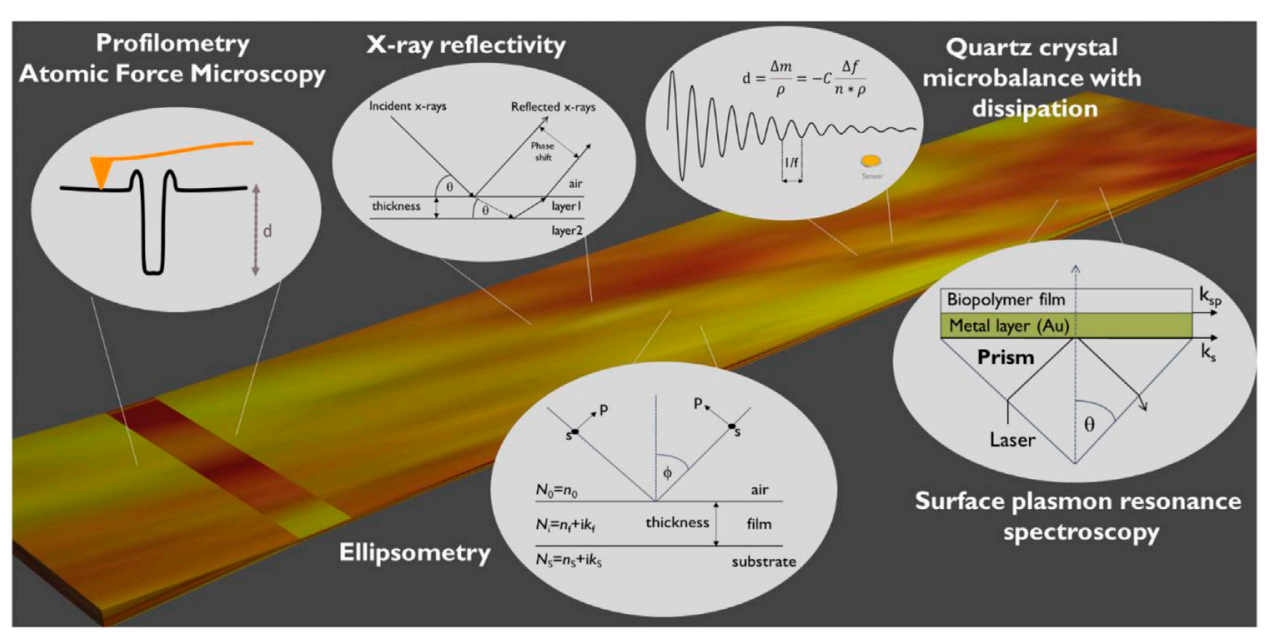

FIGURE 3 | Overview of commonly employed techniques for thickness determination of biopolymer thin films.

modeled as a homogeneous structure and lead to discrepancy with the reality. The ability to swell and film porosity as well require attention when embarking in biopolymer thin film thickness determination. Error from swelling and change in free volume is challenging to account for. For example, on the case of the methods requiring a density input, this becomes a variable. Similarly, with changing volume fraction, the refractive index is modulated. Humidity is a challenge in layer thickness determination of biopolymer thin films in general. Care needs to be taken so that values are given either at a specific humidity or over a wide range of humidity levels to provide reproducible thin film data.

\section{CONCLUSION}

Profilometry and AFM are direct methods relying on analysis of the height difference between film and substrate surface that is defined to be the film thickness. The methods are commonly applied to study dry films but can also probe wet films. Ellipsometry is the most common non-destructive method for film thickness determination and does not suffer from the threat of damaging surface as is possible when preparing an intend or scratch for the AFM and profilometry. However, ellipsometry requires information about refractive index to aid easy determination of film thickness or use of spectroscopic ellipsometry to measure on multiple wavelengths and then use of iterative fitting to output both refractive index and film thickness. The exact refractive index of many biopolymers is not available, or the literature values may be inaccurate due to variation in the

\section{REFERENCES}

Ahola, S., Salmi, J., Johansson, L.-S., Laine, J., and Österberg, M. (2008). Model Films from Native Cellulose Nanofibrils. Preparation, Swelling, and Surface Interactions. Biomacromolecules 9, 1273-1282. doi:10.1021/bm701317k

Arwin, H. (2000). Ellipsometry on Thin Organic Layers of Biological Interest: Characterization and Applications. Thin Solid Films 377-378, 48-56. doi:10.1016/s0040-6090(00)01385-7 compound structure and composition batch to batch and choice of liberation method. It seems that use of either ellipsometry or AFM/profilometry alone, leaves room for significant error and hence using several methods instead of relying on one should be considered. The surface sensitive analytics QCM-D and SPR are also rather simple techniques for thickness determination. However, it should be kept in mind that unless the values for density and refractive index increment, are known, the calculated values are estimations. The most accurate technique is probably XRR as it enables resolution in the sub-nm regime. It yields density and thickness of the layers and does not require extensive modelling of the thin films. A disadvantage, however, is the cost of an XRR system that exceeds those of, e.g., profilometry.

\section{AUTHOR CONTRIBUTIONS}

TN: Conceptualization, writing the initial draft, review and writing; SP: Conceptualization, review and writing; CP: review and writing.

\section{FUNDING}

Swedish Research Council (VR, registration number 2017-05138) and Wallenberg Wood Science Center (WWSC) are acknowledged for funding.

Aulin, C., Ahola, S., Josefsson, P., Nishino, T., Hirose, Y., Österberg, M., et al. (2009). Nanoscale Cellulose Films with Different Crystallinities and Mesostructures-Their Surface Properties and Interaction with Water. Langmuir 25, 7675-7685. doi:10.1021/la900323n Azzam, R. M. A., Bashara, N. M., and Ballard, S. S. (1978). Ellipsometry and Polarized Light. Phys. Today 31, 72. doi:10.1063/1.2994821

Buron, C. C., Membrey, F., Filiâtre, C., and Foissy, A. (2006). A New Approach to Determine the Mean Thickness and Refractive index of Polyelectrolyte Multilayer Using Optical Reflectometry. Colloids Surf., A: Physicochemical Eng. Aspects 289, 163-171. doi:10.1016/j.colsurfa.2006.04.029 
Canale, C., Torre, B., Ricci, D., and Braga, P. C. (2011). "Recognizing and Avoiding Artifacts in Atomic Force Microscopy Imaging," in Atomic Force Microscopy in Biomedical Research (Springer), 31-43. doi:10.1007/978-161779-105-5_3

Chiang, C.-J., and Yang, F.-S. (2011). P-124: Methods of Measuring Thin Film Thickness on Polymer Substrate. Dig. Tech. Pap. - Soc. Inf. Disp. Int. Symp. 42, 1570-1572. doi:10.1889/1.3621163

Craig, V. S. J., and Plunkett, M. (2003). Determination of Coupled Solvent Mass in Quartz crystal Microbalance Measurements Using Deuterated Solvents. J. Colloid Interf. Sci. 262, 126-129. doi:10.1016/s0021-9797(03) 00210-8

Cranston, E. D., and Gray, D. G. (2008). Birefringence in Spin-Coated Films Containing Cellulose Nanocrystals. Colloids Surf., A: Physicochemical Eng. Aspects 325, 44-51. doi:10.1016/j.colsurfa.2008.04.042

De Feijter, J. A., Benjamins, J., and Veer, F. A. (1978). Ellipsometry as a Tool to Study the Adsorption Behavior of Synthetic and Biopolymers at the Air-Water Interface. Biopolymers 17, 1759-1772. doi:10.1002/bip.1978.360170711

Edgar, C. D., and Gray, D. G. (2003). Smooth Model Cellulose I Surfaces from Nanocrystal Suspensions. Cellulose 10, 299-306. doi:10.1023/a: 1027333928715

Erdoğan, M., Çapan, I., Tarimci, C., and Hassan, A. K. (2008). Modeling of Vapor Sorption in Polymeric Film Studied by Surface Plasmon Resonance Spectroscopy. J. Colloid Interf. Sci 323, 235-241. doi:10.1016/j.jcis.2008.04.045

Eriksson, M., Notley, S. M., and Wågberg, L. (2007). Cellulose Thin Films: Degree of Cellulose Ordering and its Influence on Adhesion. Biomacromolecules 8, 912-919. doi:10.1021/bm061164w

Eronen, P., Junka, K., Laine, J., and Österberg, M. (2011). Interaction between Water Soluble Polysaccharides and Native Nanofibrillar Cellulose Thin Films. BioResources 6, 4200-4217.

Fält, S., Wågberg, L., Vesterlind, E.-L., and Larsson, P. T. (2004). Model Films of Cellulose II - Improved Preparation Method and Characterization of the Cellulose Film. Cellulose 11, 151-162. doi:10.1023/b:cell.0000025403.23775.75

Foster, M., Stamm, M., Reiter, G., and Huettenbach, S. (1990). X-ray Reflectometer for Study of Polymer Thin Films and Interfaces. Vacuum 41, 1441-1444. doi:10.1016/0042-207x(90)93984-q

Ganner, T., Roŝker, S., Eibinger, M., Kraxner, J., Sattelkow, J., Rattenberger, J., et al. (2015). Tunable Semicrystalline Thin Film Cellulose Substrate for HighResolution, Iin-Ssitu AFM Characterization of Enzymatic Cellulose Degradation. ACS Appl. Mater. Inter. 7, 27900-27909. doi:10.1021/ acsami.5b09948

Gesang, T., Fanter, D., Höper, R., Possart, W., and Hennemann, O.-D. (1995). Comparative Film Thickness Determination by Atomic Force Microscopy and Ellipsometry for Ultrathin Polymer Films. Surf. Interf. Anal. 23, 797-808. doi:10.1002/sia.740231202

Höök, F., Vörös, J., Rodahl, M., Kurrat, R., Böni, P., Ramsden, J. J., et al. (2002). A Comparative Study of Protein Adsorption on Titanium Oxide Surfaces Using In Situ Ellipsometry, Optical Waveguide Lightmode Spectroscopy, and Quartz crystal Microbalance/dissipation. Colloids Surf., B: Biointerfaces 24, 155-170. doi:10.1016/s0927-7765(01)00236-3

Jung, L. S., Campbell, C. T., Chinowsky, T. M., Mar, M. N., and Yee, S. S. (1998). Quantitative Interpretation of the Response of Surface Plasmon Resonance Sensors to Adsorbed Films. Langmuir 14, 5636-5648. doi:10.1021/la971228b

Kargl, R., Mohan, T., Ribitsch, V., Saake, B., Puls, J., and Stana-Kleinschek, K. (2015). Cellulose Thin Films from Ionic Liquid Solutions. Nordic Pulp Paper Res. J. 30, 6-13. doi:10.3183/npprj-2015-30-01-p006-013

Kittle, J. D., Du, X., Jiang, F., Qian, C., Heinze, T., Roman, M., et al. (2011). Equilibrium Water Contents of Cellulose Films Determined via Solvent Exchange and Quartz crystal Microbalance with Dissipation Monitoring. Biomacromolecules 12, 2881-2887. doi:10.1021/bm200352q

Kittle, J. D., Qian, C., Edgar, E., Roman, M., and Esker, A. R. (2018). Adsorption of Xyloglucan onto Thin Films of Cellulose Nanocrystals and Amorphous Cellulose: Film Thickness Effects. ACS omega 3, 14004-14012. doi:10.1021/ acsomega.8b01750

Kittle, J. D., Wang, C., Qian, C., Zhang, Y., Zhang, M., Roman, M., et al. (2012). Ultrathin Chitin Films for Nanocomposites and Biosensors. Biomacromolecules 13, 714-718. doi:10.1021/bm201631r
Kittle, J., Levin, J., and Levin, N. (2021). Water Content of Polyelectrolyte Multilayer Films Measured by Quartz crystal Microbalance and Deuterium Oxide Exchange. Sensors 21, 771. doi:10.3390/s21030771

Kontturi, E., and Spirk, S. (2019). Ultrathin Films of Cellulose: A Materials Perspective. Front. Chem. 7, 488. doi:10.3389/fchem.2019.00488

Kontturi, E., Johansson, L.-S., Kontturi, K. S., Ahonen, P., Thüne, P. C., and Laine, J. (2007). Cellulose Nanocrystal Submonolayers by Spin Coating. Langmuir 23, 9674-9680. doi:10.1021/la701262x

Kontturi, E., and Lankinen, A. (2010). Following the Kinetics of a Chemical Reaction in Ultrathin Supported Polymer Films by Reliable Mass Density Determination with X-ray Reflectivity. J. Am. Chem. Soc. 132, 3678-3679. doi:10.1021/ja100669w

Kontturi, E., Tammelin, T., and Österberg, M. (2006). Cellulose-model Films and the Fundamental Approach. Chem. Soc. Rev. 35, 1287-1304. doi:10.1039/ b601872f

Kontturi, E., Thüne, P. C., and Niemantsverdriet, J. W. (2003). Novel Method for Preparing Cellulose Model Surfaces by Spin Coating. Polymer 44, 3621-3625. doi:10.1016/s0032-3861(03)00283-0

Kontturi, K. S., Kontturi, E., and Laine, J. (2013). Specific Water Uptake of Thin Films from Nanofibrillar Cellulose. J. Mater. Chem. A. 1, 13655-13663. doi:10.1039/c3ta12998e

Kosaka, P. M., Kawano, Y., Salvadori, M. C., and Petri, D. F. S. (2005). Characterization of Ultrathin Films of Cellulose Esters. Cellulose 12, 351-359. doi:10.1007/s10570-005-2205-0

Lu, R., Zhang, X., Fu, L., Wang, H., Briber, R. M., and Wang, H. (2020). Amorphous Cellulose Thin Films. Cellulose 27, 2959-2965. doi:10.1007/s10570-02003043-7

Manini, P., Lucci, V., Lino, V., Sartini, S., Rossella, F., Falco, G., et al. (2020). Synthetic Mycomelanin Thin Films as Emergent Bio-Inspired Interfaces Controlling the Fate of Embryonic Stem Cells. J. Mater. Chem. B 8, 4412-4418. doi:10.1039/d0tb00623h

Mohan, T., Niegelhell, K., Zarth, C. S. P., Kargl, R., Köstler, S., Ribitsch, V., et al. (2014). Triggering Protein Adsorption on Tailored Cationic Cellulose Surfaces. Biomacromolecules 15, 3931-3941. doi:10.1021/bm500997s

Mohan, T., Spirk, S., Kargl, R., Doliška, A., Ehmann, H. M. A., Köstler, S., et al. (2012). Watching Cellulose Grow - Kinetic Investigations on Cellulose Thin Film Formation at the Gas-Solid Interface Using a Quartz crystal Microbalance with Dissipation (QCM-D). Colloids Surf., A: Physicochemical Eng. Aspects 400, 67-72. doi:10.1016/j.colsurfa.2012.02.053

Mykhaylyk, T. A., Dmitruk, N. L., Evans, S. D., Hamley, I. W., and Henderson, J. R. (2007). Comparative Characterisation by Atomic Force Microscopy and Ellipsometry of Soft and Solid Thin Films. Surf. Interf. Anal. 39, 575-581. doi:10.1002/sia.2566

Niegelhell, K., Leimgruber, S., Grießer, T., Brandl, C., Chernev, B., Schennach, R., et al. (2016a). Adsorption Studies of Organophosphonic Acids on Differently Activated Gold Surfaces. Langmuir 32, 1550-1559. doi:10.1021/ acs.langmuir.5b04467

Niegelhell, K., Süßenbacher, M., Jammernegg, K., Ganner, T., Schwendenwein, D., Schwab, H., et al. (2016b). Enzymes as Biodevelopers for Nano- and Micropatterned Bicomponent Biopolymer Thin Films. Biomacromolecules 17, 3743-3749. doi:10.1021/acs.biomac.6b01263

Niegelhell, K., Süßenbacher, M., Sattelkow, J., Plank, H., Wang, Y., Zhang, K., et al. (2017). How Bound and Free Fatty Acids in Cellulose Films Impact Nonspecific Protein Adsorption. Biomacromolecules 18, 4224-4231. doi:10.1021/ acs.biomac.7b01260

Niinivaara, E., Faustini, M., Tammelin, T., and Kontturi, E. (2016). Mimicking the Humidity Response of the Plant Cell Wall by Using Two-Dimensional Systems: The Critical Role of Amorphous and Crystalline Polysaccharides. Langmuir 32, 2032-2040. doi:10.1021/acs.langmuir.5b04264

Norgren, M., Notley, S. M., Majtnerova, A., and Gellerstedt, G. (2006). Smooth Model Surfaces from Lignin Derivatives. I. Preparation and Characterization. Langmuir 22, 1209-1214. doi:10.1021/la052284c

Orelma, H., Filpponen, I., Johansson, L.-S., Laine, J., and Rojas, O. J. (2011). Modification of Cellulose Films by Adsorption of CMC and Chitosan for Controlled Attachment of Biomolecules. Biomacromolecules 12, 4311-4318. doi:10.1021/bm201236a 
Palasingh, C., Ström, A., Amer, H., and Nypelö, T. (2021). Oxidized Xylan Additive for Nanocellulose Films - A Swelling Modifier. Int. J. Biol. Macromolecules 180, 753-759. doi:10.1016/j.ijbiomac.2021.03.062

Peresin, M. S., Kammiovirta, K., Setälä, H., and Tammelin, T. (2012). Structural Features and Water Interactions of Etherified Xylan Thin Films. J. Polym. Environ. 20, 895-904. doi:10.1007/s10924-012-0469-7

Peterlinz, K. A., and Georgiadis, R. (1996). Two-color Approach for Determination of Thickness and Dielectric Constant of Thin Films Using Surface Plasmon Resonance Spectroscopy. Opt. Commun. 130, 260-266. doi:10.1016/0030-4018(96)00238-6

Puspasari, T., Pradeep, N., and Peinemann, K.-V. (2015). Crosslinked Cellulose Thin Film Composite Nanofiltration Membranes with Zero Salt Rejection. J. Membr. Sci. 491, 132-137. doi:10.1016/j.memsci.2015.05.002

Raghuwanshi, V. S., and Garnier, G. (2019). Cellulose Nano-Films as BioInterfaces. Front. Chem. 7, 535. doi:10.3389/fchem.2019.00535

Reid, M. S., Villalobos, M., and Cranston, E. D. (2016). Cellulose Nanocrystal Interactions Probed by Thin Film Swelling to Predict Dispersibility. Nanoscale 8, 12247-12257. doi:10.1039/c6nr01737a

Reviakine, I., Johannsmann, D., and Richter, R. P. (2011). Hearing what You Cannot See and Visualizing what You Hear: Interpreting Quartz crystal Microbalance Data from Solvated Interfaces. Anal. Chem. 83 (23), 8838-8848.

Sampl, C., Niegelhell, K., Reishofer, D., Resel, R., Spirk, S., and Hirn, U. (2019). Multilayer Density Analysis of Cellulose Thin Films. Front. Chem. 7, 251. doi:10.3389/fchem.2019.00251

Sauerbrey, G. n. (1959). Verwendung von Schwingquarzen zur Wägung dünner Schichten und zur Mikrowägung. Z. Physik 155, 206-222. doi:10.1007/ bf01337937

Schaub, M., Wenz, G., Wegner, G., Stein, A., and Klemm, D. (1993). Ultrathin Films of Cellulose on Silicon Wafers. Adv. Mater. 5, 919-922. doi:10.1002/ adma.19930051209

Siderov, V., Mladenova, D., Yordanov, R., Milenkov, V., Ohlidal, M., Salyk, O., et al. (2013). Film Thickness Measurement by Optical Profilometer MicroProf ${ }^{\circledR}$ FRT. Bulgarian Chem. Commun. 45, 194-197.

Siqueira, D. F., Koehler, K., and Stamm, M. (1995). Structures at the Surface of Dry Thin Films of Grafted Copolymers. Langmuir 11, 3092-3096. doi:10.1021/ la00008a039

Spirk, S., Findenig, G., Doliska, A., Reichel, V. E., Swanson, N. L., Kargl, R., et al. (2013). Chitosan-silane Sol-Gel Hybrid Thin Films with Controllable Layer Thickness and Morphology. Carbohydr. Polym. 93, 285-290. doi:10.1016/ j.carbpol.2012.04.030

Sun, L., Svedhem, S., and Åkerman, B. (2014). Construction and Modeling of Concatemeric DNA Multilayers on a Planar Surface as Monitored by QCM-D and SPR. Langmuir 30, 8432-8441. doi:10.1021/la500716d

Tammelin, T., Abburi, R., Gestranius, M., Laine, C., Setälä, M., and Österberg, fnm. (2015). Correlation between Cellulose Thin Film Supramolecular Structures and Interactions with Water. Soft Matter 11, 4273-4282. doi:10.1039/ c5sm00374a
Tammelin, T., Österberg, M., Johansson, L.-S., and Laine, J. (2006). Preparation of Lignin and Extractive Model Surfaces by Using Spincoating Technique Application for QCM-D Studies. Nordic Pulp Paper Res. J. 21, 444-450. doi:10.3183/npprj-2006-21-04-p444-450

Tengvall, P., Lundström, I., and Liedberg, B. (1998). Protein Adsorption Studies on Model Organic Surfaces: an Ellipsometric and Infrared Spectroscopic Approach. Biomaterials 19, 407-422. doi:10.1016/s0142-9612(97)00110-5

Thomas, R. K., and Penfold, J. (1996). Neutron and X-ray Reflectometry of Interfacial Systems in Colloid and Polymer Chemistry. Curr. Opin. Colloid Interf. Sci. 1, 23-33. doi:10.1016/s1359-0294(96)80040-9

Ton-That, C., Shard, A. G., and Bradley, R. H. (2000). Thickness of Spin-Cast Polymer Thin Films Determined by Angle-Resolved XPS and AFM Tip-Scratch Methods. Langmuir 16, 2281-2284. doi:10.1021/la990605c

Voinova, M. V., Rodahl, M., Jonson, M., and Kasemo, B. (1999). Viscoelastic Acoustic Response of Layered Polymer Films at Fluid-Solid Interfaces: Continuum Mechanics Approach. Phys. Scr. 59, 391-396. doi:10.1238/ physica.regular.059a00391

Weißl, M., Hobisch, M. A., Johansson, L. S., Hettrich, K., Kontturi, E., Volkert, B., et al. (2019a). Cellulose Carbamate Derived Cellulose Thin Films: Preparation, Characterization and Blending with Cellulose Xanthate. Cellulose 26, 7399-7410. doi:10.1007/s10570-019-02600-z

Weißl, M., Niegelhell, K., Reishofer, D., Zankel, A., Innerlohinger, J., and Spirk, S. (2018). Homogeneous Cellulose Thin Films by Regeneration of Cellulose Xanthate: Properties and Characterization. Cellulose 25, 711-721. doi:10.1007/s10570-017-1576-3

Weißl, M., Rath, T., Sattelkow, J., Plank, H., Eyley, S., Thielemans, W., et al. (2019b). Multi-layered Nanoscale cellulose/CuInS2 sandwich Type Thin Films. Carbohydr. Polym. 203, 219-227. doi:10.1016/j.carbpol.2018.09.063

Conflict of Interest: The authors declare that the research was conducted in the absence of any commercial or financial relationships that could be construed as a potential conflict of interest.

Publisher's Note: All claims expressed in this article are solely those of the authors and do not necessarily represent those of their affiliated organizations, or those of the publisher, the editors and the reviewers. Any product that may be evaluated in this article, or claim that may be made by its manufacturer, is not guaranteed or endorsed by the publisher.

Copyright (c) 2021 Spirk, Palasingh and Nypelö. This is an open-access article distributed under the terms of the Creative Commons Attribution License (CC BY). The use, distribution or reproduction in other forums is permitted, provided the original author(s) and the copyright owner(s) are credited and that the original publication in this journal is cited, in accordance with accepted academic practice. No use, distribution or reproduction is permitted which does not comply with these terms. 